\section{Alnylam's RNAi therapy targets amyloid disease}

The most clinically advanced RNA interference (RNAi) therapeutic achieved a milestone in April when Alnylam Pharmaceuticals in Cambridge, Massachusetts, reported positive results for patisiran, a small interfering RNA (siRNA) oligonucleotide targeting transthyretin for treating familial amyloidotic polyneuropathy (FAP). Alnylam had earlier reported that patisiran (ALN-TTRO2) could reduce circulating transthyretin levels by $82-87 \%$ compared with placebo (N. Engl. J. Med. $369,819-829,2013$ ) in a phase 1 study. With the new phase 2 data, "for the first time it appears that one can connect the dots from

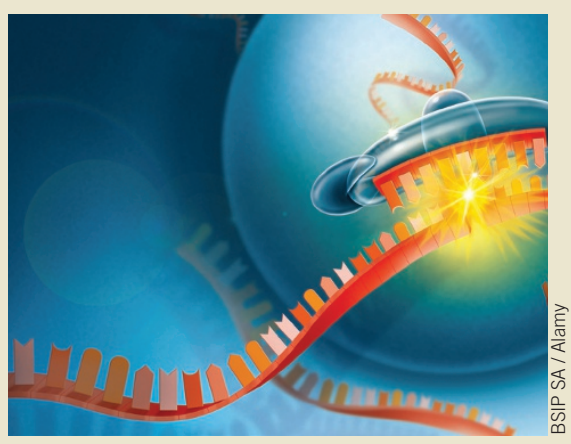

RNA interference's silencing of target genes could result in potent therapeutics. knocking down the target to potentially seeing a change in clinical measures," says Michael Schmidt, an analyst for the investment bank Leerink Partners of Boston.

In FAP, also called transthyretin-related hereditary amyloidosis, there is a an abnormal buildup of hepatocyte-derived amyloid protein in various organs and tissues, especially in the peripheral nervous system, which causes a progressive polyneuropathy. The open-label trial extension results presented at the American Academy of Neurology annual meeting in Washington, DC, on April 18-25 showed a mean 2.5\% improvement in neurologic function score at 12 months compared with a $13-18 \%$ deterioration for historical controls. The caveat is that the results only included 20 patients and there was no true control group. "It looks like these patients in the phase 2 are doing better than expected," said Angela Dispenzieri, an amyloidosis specialist at the Mayo Clinic in Rochester, Minnesota, adding that the ongoing phase 3 study will provide a fairer comparison.

Since the discovery of siRNA in mammals in 2001, the ability to turn siRNA into drugs for controlling gene expression has been hampered by delivery obstacles. Companies have tried dozens of modifications to stop siRNA pieces from being rapidly degraded by nucleases in circulation. It has also proved difficult to formulate the siRNA agents to efficiently penetrate cells for mRNA target degradation (Nat. Biotechnol. 30, 1154-1157, 2012). Patisiran siRNA targets a conserved sequence in the $3^{\prime}$ untranslated region of nonmutant and mutant mRNA for transthyretin. The siRNAs are enclosed in lipid nanoparticles (LNPS) and delivered every three weeks intravenously. But LNPs are an interim solution. "This one isn't ideal," says Alan Carr, an analyst at Needham \& Company of New York. Alnylam's newer transthyretin-targeting revusiran non-LNP siRNA, now in phase 2 for familial amyloidotic cardiomyopathy, is further engineered for nuclease resistance and is linked to a glycoprotein that is taken up by the liver, where most transthyretin is produced. Even more robust are the latest clinical-stage molecules, which could be delivered subcutaneously as seldom as every three months, says Carr.

So far, patisiran appears to be safe, but vision impairment could be a concern. This is because transthyretin is a carrier of vitamin A, needed for converting light stimuli into neuronal signaling by the retina. "There are many other transport systems with vitamin $A$, so vitamin A deficiency is not a major concern," says Alnylam's president and COO Barry Greene. "However, we're monitoring for it."

Although FAP is a rare disease, the buildup of abnormal amyloid deposits in the heart in familial amyloidotic cardiomyopathy, is four times more prevalent, and there is even more market potential in senile systemic amyloidosis. There's "an order of magnitude more patients who can potentially benefit from these drugs," says Dispenzieri.

In the meantime, patisiran and revusiran must succeed in phase 3 and show superiority to a transthyretin-targeting antisense oligonucleotide from Isis Pharmaceuticals in Carlsbad, California, also in phase 3, and to New York-based Pfizer's transthyretin stabilizer tafamidis (Vyndaqel), already approved in Europe (Nat. Biotechnol. 30, 121, 2012). But if Alnylam finally has the right delivery platform, applications could quickly multiply. The company is targeting at least a dozen other liver-produced disease proteins. "You're using the same type of molecule, for each," says Carr. "That makes drug development easier for Alnylam, relative to small-molecule drug development."

Ken Garber Ann Arbor, Michigan

\section{Backlog cripples China's drug regulator}

Unprocessed applications are clogging up China's Food and Drug Administration, delaying the arrival of new drugs to the market, according to a recent report. Chinese drug developers and subsidiaries of multinational pharma companies have long complained about the country's slow drug approval process. But a severe lack of staffing has left the Center for Drug Evaluation of the CFDA with a backlog of applications topping 18,000 at the end of 2014 , an increase of more than 4,000 since the end of the previous year. Staff shortages engender problems, such as improper, repetitive applications for generic drugs, and occasionally corruption. Another critical factor in the delay is that drug reviewers carry personal responsibility for their professional decisions with potentially serious consequences for mistakes, intended or not (Nat. Biotechnol. 25, 835-837, 2007). As a result, reviewers must seek supervisory approval for each decision. The CFDA said in January that it plans to reduce the backlog in the next three years by adding personnel and funding, streamlining relevant mechanisms and increasing the speed of review.

\section{Brazil approves transgenic eucalyptus}

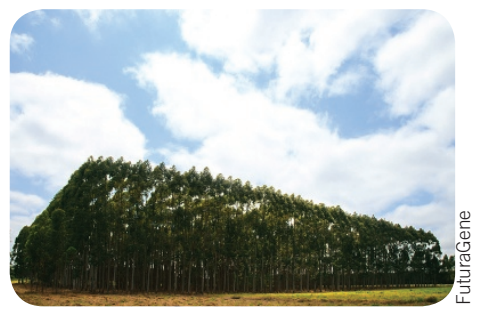

FuturaGene's eucalyptus

FuturaGene's genetically modified (GM) eucalyptus was approved for commercial release by the Brazilian National Technical Commission on Biosafety in April. It is the first GM eucalyptus approved worldwide. FuturaGene, which merged with Rehovot, Israel's CBD in 2006, and is now wholly owned by São Paulo, Brazil-based Suzano Pulp and Paper, the second largest producer of eucalyptus pulp globally. FuturaGene says the GM tree produces $20 \%$ more wood compared with its conventional variety Eucalyptus spp. and is ready for harvest in five and a half years instead of seven. FuturaGene inserted into eucalyptus a gene encoding an Arabidopsis thaliana protein that facilitates cell-wall expansion and accelerates growth. A month earlier, in March, FuturaGene's facilities in Brazil were vandalized by approximately 1,000 activists representing Brazil's Landless Workers' Movement. They destroyed GM eucalyptus seedlings in an action coinciding with a meeting held by the National Technical Commission on Biosafety in Brasilia. 